\title{
How to Choose a President, Mayor, Chair: Balinski and Laraki Unpacked
}

\author{
Harrie de Swart (i)
}

It is the (main) task of philosophy to show that things do not have to be the way we are used to, that they might be different and that in some cases they should be different! - Johan de Iongh

7 $\mathrm{n}$ their book [2] and their articles [3-6] — some of them pretty long-Michel Balinski and Rida Laraki present a new voting method, called majority judgment, which has many interesting properties and in many cases yields different outcomes from the more familiar voting methods. The main aim of this paper is to give the reader an easily accessible, compact, and vivid exposition of the most important results by these authors.

In most Western European countries, plurality rule (PR) is used in elections. Voters are to choose the candidate (or party) that they most prefer. The result is called a profile $p$, which-from a mathematical point of view-is a function that assigns to every voter $i$ his or her most preferred candidate $p(i)$. Plurality rule aggregates these individual choices, as rendered in a profile $p$, into a social ranking $\succeq_{\mathrm{PR}(p)}$ of the candidates, defined as follows: $x \succeq_{\mathrm{PR}(p)} y$ means that the number of voters in $p$ who most prefer $x$ is greater than or equal to the number of voters in $p$ who most prefer $y$.

In some countries, such as Ireland and Malta, voters are asked to give a ranking of the candidates. The result is again called a profile, but now - from a mathematical perspective-a profile $p$ is a function that assigns to every voter $i$ a (linear) ordering $p(i)$ of the candidates. Majority rule (MR) assigns to every profile $p$ a social relation $\succeq_{\mathrm{MR}(p)}$ of the candidates, defined as follows: $x \succeq_{\mathrm{MR}(p)} y$ means that the number of voters in $p$ who prefer $x$ to $y$ is greater than or equal to the number of voters in $p$ who prefer $y$ to $x$. We shall write $\succeq_{\mathrm{PR}}$ and $\succeq_{\mathrm{MR}}$ when the profile $p$ is clear from the context.

It is easy to see that in the case of only two candidates, majority rule and plurality rule coincide.

In 2010, Balinski and Laraki [2] made clear that there is an electoral rule with nice properties, called majority judgment (MJ), that results from asking voters for an evaluation of the candidates instead of their preference orderings over the candidates. Possible evaluations are, for example, excellent (ex), very good (vg), good (go), acceptable (ac), poor (po), and reject (re). The number of possible evaluations should be sufficiently large so that a voter is able to express a distinction between the candidates. That is, if one wants to distinguish two candidates, then one is able to express this.

Assuming that this language of grades is sufficiently rich, evaluations are much more informative than orderings of preference: from the evaluations of the candidates by a voter, one can deduce the ordering of preference, but not vice versa. If three voters say that they prefer candidate $A$ to candidate $B$, they can mean totally different things: the first voter that he finds $A$ good and $B$ slightly less good, the second that she finds $A$ excellent and $B$ bad, and the third that she finds $A$ bad and $B$ even worse. The result is a Babylonian confusion of tongues: different people produce the same words, but they may all mean something different. Already in the first half of the last century, Gerrit Mannoury, Luitzen Egbertus Jan Brouwer, David van 
Dantzig, Frederik van Eeden, among others, united in the Signific Circle, stressed the importance of a careful and accurate use of language in order to prevent such a Babylonian confusion of tongues; see [14, Section 7.3].

What Is Wrong with Plurality and Majority Rule?

Majority rule cannot take into account the intensities with which voters prefer one candidate to the other. In 2016, Balinski and Laraki [3] made this somewhat vague observation precise for the first time: Consider two candidates $A$ and $B$ who receive the evaluations below from five voters, as shown in the following opinion profile.

\begin{tabular}{l|lllll} 
& \multicolumn{1}{|c}{$\mathbf{1}$} & $\mathbf{2}$ & \multicolumn{1}{c}{$\mathbf{3}$} & \multicolumn{1}{c}{$\mathbf{4}$} & \multicolumn{1}{c}{$\mathbf{5}$} \\
\hline$A$ & go(od) & ac(ceptable) & po(or) & ex(cellent) & v(ery)g(ood) \\
& $\uparrow$ & $\uparrow$ & $\uparrow$ & $\downarrow$ & $\downarrow$ \\
$B$ & v(ery)g(ood) & go(od) & ac(ceptable) & po(or) & re(ject)
\end{tabular}

The first three voters slightly prefer $B$ to $A$, while the last two voters strongly prefer $A$ to $B$. Under majority rule, in this case identical to plurality rule, $B$ is the "Condorcet winner"; i.e., $B$ beats every other candidate in a pairwise comparison. In our example, $B$ beats $A$ with 3 against 2: $B \succ_{\mathrm{MR}} A$.

However, if we look at the evaluations of $A$ and $B$, ordered from high to low, then from the above opinion profile, the following merit profile emerges:

\begin{tabular}{l|lllll}
$A$ & ex(cellent) & v(ery)g(ood) & go(od) & ac(ceptable) & po(or) \\
$B$ & v(ery)g(ood) & go(od) & ac(ceptable) & po(or) & re(ject)
\end{tabular}

It is $A$ that has the better ratings. In other words, the evaluations of $A$ dominate those of $B$. Therefore, majority judgment will designate $A$ as the winner: $A \succ_{\mathrm{MJ}} B$.

Since $B \succ_{\mathrm{MR}} A$, it follows that majority rule does not respect dominance, and one may argue that any reasonable electoral method should respect dominance.

We make the following definition.

Definition 1. The merit profile $\alpha=\left(\alpha_{1}, \alpha_{2}, \ldots, \alpha_{n}\right)$ of candidate $A$ dominates merit profile $\beta=\left(\beta_{1}, \beta_{2}, \ldots, \beta_{n}\right)$ of candidate $B$ if for every $i, \alpha_{i} \geq \beta_{i}$, and for at least one $k$, $\alpha_{k}>\beta_{k}$.

\section{Majority Judgment}

Let us consider again the merit profile just given:

\begin{tabular}{l|lllllllll}
$A$ & $\mathrm{ex}$ & $\rightarrow$ & $\mathrm{vg}$ & $\rightarrow$ & go & $\leftarrow$ & ac & $\leftarrow$ & po \\
$B$ & $\mathrm{vg}$ & $\rightarrow$ & $\mathrm{go}$ & $\rightarrow$ & ac & $\leftarrow$ & po & $\leftarrow$ & re
\end{tabular}

There is a three-fifths majority that thinks $A$ deserves at least a rating of go(od), and there is another three-fifths majority that judges that $A$ deserves at most go(od). For this reason, we say that the "majority grade" of $A$ is good by definition. The majority grade of $B$ is only ac(ceptable). Because $A$ has a higher majority grade than $B$, majority judgment (MJ) will $\operatorname{rank} A$ above $B: A \succ_{\mathrm{MJ}} B$. This order is the logical result of majorities that decide on the evaluations of the candidates. Majority judgment (MJ) measures the electorate's support for the candidates and orders them proportionately. With majority rule (MR), voters cannot express their evaluations about the candidates; each voter is limited to supporting one candidate to the exclusion of all others.

Majority rule (MR) looks vertically at majorities in the following opinion profile:

\begin{tabular}{c|ccccc} 
& $\mathbf{1}$ & $\mathbf{2}$ & $\mathbf{3}$ & $\mathbf{4}$ & $\mathbf{5}$ \\
\hline$A$ & go & ac & po & ex & vg \\
& $\uparrow$ & $\uparrow$ & $\uparrow$ & $\downarrow$ & $\downarrow$ \\
$B$ & vg & go & ac & po & re
\end{tabular}

with $B \succ_{\text {MR }} A$ with a vote of 3 to 2 , while majority judgment (MJ) looks horizontally at majorities in the merit profile:

\begin{tabular}{c|ccccc} 
& $\mathbf{1}$ & $\mathbf{2}$ & $\mathbf{3}$ & $\mathbf{4}$ & $\mathbf{5}$ \\
\hline$A$ & $\mathrm{ex} \rightarrow$ & $\mathrm{vg} \rightarrow$ & go & $\leftarrow \mathrm{ac}$ & $\leftarrow \mathrm{po}$ \\
$B$ & $\mathrm{vg} \rightarrow$ & $\mathrm{go} \rightarrow$ & ac & $\leftarrow \mathrm{po}$ & $\leftarrow \mathrm{re}$
\end{tabular}

with $A \succ_{\mathrm{MJ}} B$, good versus acceptable. Majority judgment (MJ) respects dominance; majority rule (MR) does not.

Clearly, every opinion profile generates a merit profile, but notice that the same merit profile may result from different opinion profiles. For instance, the following opinion profile, different from the one above, will result in the same merit profile:

\begin{tabular}{l|lllll} 
& $\mathbf{1}$ & $\mathbf{2}$ & $\mathbf{3}$ & $\mathbf{4}$ & $\mathbf{5}$ \\
\hline$A$ & vg & ex & go & ac & po \\
& $\downarrow$ & $\downarrow$ & $\downarrow$ & $\uparrow$ & $\uparrow$ \\
$B$ & po & re & ac & go & vg
\end{tabular}

For this opinion profile we have $A \succ_{\mathrm{MR}} B$, because $A$ beats $B$ with 3 against 2 .

What if two candidates $B$ and $C$ have the same majority grade?

\begin{tabular}{l|lllll}
$B$ & vg $\rightarrow$ & {$[$ go $\rightarrow$} & ac & $\leftarrow$ po $]$ & $\leftarrow r e$ \\
$C$ & vg $\rightarrow$ & {$[$ go $\rightarrow$} & ac & $\leftarrow r e]$ & $\leftarrow r e$
\end{tabular}

Both $B$ and $C$ have a three-fifths majority for [ac, ac]; in other words, the majority grade of both $B$ and $C$ is acceptable. In such a case, we will look at four-fifths majorities. Here $B$ has a four-fifths majority for [go, po]: there is a four-fifths majority that thinks that $B$ deserves at least a rating of poor, and there is another four-fifths majority that judges that $B$ deserves at most a rating of good. However, $C$ has a four-fifths majority for [go, re]. Because [go, po] dominates [go, re], the four-fifths majority of $B$ dominates that of $C$, and hence by definition, $B \succ_{\mathrm{MJ}} C$. 
What if the four-fifths majority of $B$ differs from the fourfifths majority of $C$ but does not dominate it? Consider the following example:

$$
\begin{array}{l|lllll}
B & \mathrm{vg} \rightarrow & {[\mathrm{go} \rightarrow} & \text { ac } & \leftarrow \mathrm{po}] & \leftarrow \mathrm{re} \\
C^{\prime} & \mathrm{vg} \rightarrow & {[\mathrm{vg} \rightarrow} & \text { ac } & \leftarrow \mathrm{re}] & \leftarrow \mathrm{re}
\end{array}
$$

The four-fifths majority for $B$ is [go, po], while the fourfifths majority of $C$ is $[\mathrm{vg}, \mathrm{re}]$. Neither of these four-fifths majorities dominates the other. Because $\mathrm{v}($ ery $) \mathrm{g}(\mathrm{ood}) \succ$ go(od) $\succcurlyeq \operatorname{po}($ or $) \succ$ re(ject), in other words, because the four-fifths majority interval [go, po] for $B$ is sharply contained within the four-fifths majority interval [vg, re] for $C$, we say that there is more consensus in the four-fifths majority interval for $B$ than in the four-fifths majority interval for $C$ and declare that majority judgment will rank the candidate with more consensus in the four-fifths majority interval higher than the other one. Therefore, by definition, $B \succ_{\mathrm{MJ}} C^{\prime}$.

Definition 2. (MJ ranking) Let $A$ 's grades be $\alpha=$ $\left(\alpha_{1}, \ldots, \alpha_{n}\right)$ and let $B$ 's grades be $\beta=\left(\beta_{1}, \ldots, \beta_{n}\right)$, both written from highest to lowest. Suppose the most accurate majority for which the candidates differ is the $\frac{n-k+1}{n}$ majority for $\left[\alpha_{k}, \alpha_{n-k+1}\right] \neq\left[\beta_{k}, \beta_{n-k+1}\right]$. For instance, in the example at the beginning of this section, $n=5, k=2$, and so $n-k+1=4$

Let $A \succ_{\mathrm{MJ}} B$ mean that $A$ 's block $\left[\alpha_{k}, \ldots, \alpha_{n-k+1}\right]$ dominates $B$ 's block $\left[\beta_{k}, \ldots, \beta_{n-k+1}\right]$, or $A$ 's block is more consensual than $B$ 's. In other words, $A \succ_{\mathrm{MJ}} B:=\alpha_{k} \succeq \beta_{k}$ and $\alpha_{n-k+1} \succeq \beta_{n-k+1}$, with at least one $\succeq$ strict, or $\beta_{k} \succ \alpha_{k} \succeq \alpha_{n-k+1} \succ \beta_{n-k+1}$. Otherwise, their sets of grades are identical, and $A \approx_{\mathrm{MJ}} B$.

Let $A \succeq_{\mathrm{MJ}} B$ mean that $A \succ_{\mathrm{MJ}} B$ or $A \approx_{\mathrm{MJ}} B$. From this definition, it follows that $\succeq_{\mathrm{MJ}}$ is transitive, i.e., if $A \succeq_{\mathrm{MJ}} B$ and $B \succeq_{\mathrm{MJ}} C$, then $A \succeq_{\mathrm{MJ}} C$.

\section{American Presidential Election 2016}

Balinski and Laraki describe in [4] an experiment with a limited number of voters in the context of the United States presidential election in 2016, illustrating that also in practice, one and the same merit profile may result from different opinion profiles and in this way explaining how Trump instead of Clinton could win against all expectations.

In a poll among 1787 voters by the Pew Research Center in March 2016, Clinton's ratings dominated those of Trump:

\begin{tabular}{l|llllll} 
& great & good & average & poor & terrible & majority grade \\
\hline Clinton & $11 \%$ & $22 \%$ & $\mathbf{2 0} \%$ & $16 \%$ & $31 \%$ & av \\
Trump & $10 \%$ & $16 \%$ & $12 \%$ & $\mathbf{1 5 \%}$ & $47 \%$ & po
\end{tabular}

As pointed out by Balinski and Laraki, the majority grade of Clinton is average: there is an $11 \%+22 \%+20 \%=53 \%$ majority of voters who think that Clinton deserves at least a grade of average, and there is another $31 \%+16 \%+20 \%=$
$67 \%$ majority of voters who judge that she deserves at most a grade of average. The majority grade of Trump is poor. Hence majority judgment (MJ) predicts that Clinton will be the winner: Clinton $\succ_{\mathrm{MJ}}$ Trump.

But Balinski and Laraki go on to say that majority rule (MR) can easily fail to designate Clinton the prospective winner. Namely, assume that the above merit profile for Clinton and Trump is based on the following opinion profile of these candidates:

\begin{tabular}{l|ccccccccc} 
& $\mathbf{1 0}$ & $\mathbf{1 6}$ & $\mathbf{1 2}$ & $\mathbf{1 5}$ & $\mathbf{1 6}$ & $\mathbf{1 1}$ & $\mathbf{1 2}$ & $\mathbf{0 4}$ & $\mathbf{0 4}$ \\
\hline Clinton & go & av & po & te & te & gr & go & av & po \\
& $\uparrow$ & $\uparrow$ & $\uparrow$ & $\uparrow$ & & $\downarrow$ & $\downarrow$ & $\downarrow$ & $\downarrow$ \\
Trump & gr & go & av & po & te & te & te & te & te
\end{tabular}

This opinion profile is in accord with the above merit profile. For example, the $22 \%$ of voters who gave Clinton a rating of good are now divided into two groups: a $10 \%$ group that rates Clinton as good and Trump as great and a group of $12 \%$ of voters who rate Clinton as good and Trump as terrible. In particular, $10 \%+16 \%+12 \%+$ $15 \%=53 \%$ prefer Trump (slightly) to Clinton, while $11 \%+$ $12 \%+4 \%+4 \%=31 \%$ prefer Clinton (strongly) to Trump. So majority rule (MR) makes Trump the winner: Trump $\succ_{\mathrm{MR}}$ Clinton.

\section{French Presidential Elections in 2017}

To illustrate that majority judgment can be applied in the case of large electorates, Balinski and Laraki did many experiments with majority judgment in the context of the French presidential elections.

They report in [2, Section 2.6] for the years 2002 and 2007, while in [3], they analyze the French presidential elections of 2012.

There are two rounds of voting: If a candidate receives more than half of the votes cast in the first round, he is elected. If not, there is a second round between the two candidates with the most votes in the first round.

In the presidential election of April 23, 2017, François Hollande, Nicolas Sarkozy, and Marine Le Pen were initially the most important candidates. Suppose the orderings of preference were as follows:

\begin{tabular}{l|lll}
$34 \%$ & Hollande & Sarkozy & Le Pen \\
$32 \%$ & Sarkozy & Hollande & Le Pen \\
$34 \%$ & Le Pen & Sarkozy & Hollande
\end{tabular}

In the second round, Sarkozy is eliminated with the least number of votes, and Hollande receives 34\%+32\% $=66 \%$ of the votes and wins. Now suppose Hollande receives more support in the first round at the expense of Le Pen:

\begin{tabular}{l|lll}
$34 \%+3 \%=37 \%$ & Hollande & Sarkozy & Le Pen \\
$32 \%$ & Sarkozy & Hollande & Le Pen \\
$34 \%-3 \%=31 \%$ & Le Pen & Sarkozy & Hollande
\end{tabular}

Then Le Pen does not participate in the second round, which Sarkozy wins with $32 \%+31 \%=63 \%$ of the vote. More support for Hollande (in the first round) means losing 
instead of winning (in the second round). That is, the French electoral system is not monotonic.

Balinski and Laraki [5] also point out that for each of the three candidates, there was a large majority of French voters who opposed his or her candidacy. Why did they participate (initially)? Because plurality rule (PR) in two rounds gives them the chance to qualify for the second round against Le Pen with approximately $20 \%$ of the votes in the first round-history tells us that this usually is enough to qualify for the second round-and to win in the second round. It is ironic that three candidates each rejected by a large majority have monopolized the debate for such a long time.

The following analysis of the French presidential elections in 2017 is due to the present author. In the presidential election of April 23, 2017, Emmanuel Macron, Marine Le Pen, and François Fillon achieved the highest percentages in the first round, in that order ("Actual" column below):

\begin{tabular}{l|ll} 
& Actual & Hypothetical \\
\hline Macron & $24.01 \%$ & $25.31 \%$ \\
Le Pen & $21.30 \%$ & $20.00 \%$ \\
Fillon & $20.01 \%$ & $20.01 \%$
\end{tabular}

If Macron had received $1.30 \%$ more support in the first round at the expense of Le Pen ("Hypothetical" column), then the second round would have been between Macron and Fillon, and Fillon might have become president instead of Macron. Thus more support in the first round may mean that you lose in the second round! In other words, the French electoral system is not monotonic.

La Fabrique Spinoza performed a survey on April 11-12, 2017, among one thousand people who were deemed to be representative of the French population. This poll provided the following results: ${ }^{1}$

\begin{tabular}{l|cccccc} 
& très bien & bien & assez bien & passable & insuffisant & à rejeter \\
\hline Mélenchon & $13.3 \%$ & $22.2 \%$ & $\mathbf{2 8 . 8} \%$ & $9.1 \%$ & $11.1 \%$ & $15.5 \%$ \\
Macron & $9.6 \%$ & $22.8 \%$ & $\mathbf{2 5 . 7} \%$ & $11.0 \%$ & $11.1 \%$ & $19.8 \%$ \\
Hamon & $5.5 \%$ & $11.7 \%$ & $29.4 \%$ & $\mathbf{1 7 . 0} \%$ & $15.3 \%$ & $21.1 \%$ \\
Dupont- & $3.9 \%$ & $9.5 \%$ & $23.9 \%$ & $\mathbf{1 7 . 9} \%$ & $20.4 \%$ & $24.4 \%$ \\
$\quad$ Aignan & & & & & & \\
Le Pen & $15.3 \%$ & $14.0 \%$ & $16.3 \%$ & $\mathbf{6 . 7} \%$ & $13.6 \%$ & $34.1 \%$ \\
$\ldots$ & & & & & &
\end{tabular}

Mélenchon's majority rating is assez bien: a majority of $13.3 \%+22.2 \%+28.8 \%=64.3 \%$ believe that he deserves at least assez bien, and another majority of $15.5 \%+$ $11.1 \%+09.1 \%+28.8 \%=64.5 \%$ believe that he deserves at most assez bien. In a similar way, we see that Macron's majority rating is assez bien, while the majority ratings of Hamon, Dupont-Aignan, and Le Pen are all passable. The majority ratings of the various candidates are indicated in the table above with boldface. How does majority judgment (MJ) order Mélenchon and Macron?

\begin{tabular}{l|cccccc} 
& $\begin{array}{c}\text { très } \\
\text { bien }\end{array}$ & bien & assez bien & passable & insuffisant & $\begin{array}{c}\text { à } \\
\text { rejeter }\end{array}$ \\
\hline Mélenchon & $13.3 \%$ & $22.2 \%$ & $14.5+$ & $9.1 \%$ & $11.1 \%$ & $15.5 \%$ \\
& & & $14.3 \%$ & & & \\
Macron & $9.6 \%$ & $22.8 \%$ & $17.6+8.1 \%$ & $11.0 \%$ & $11.1 \%$ & $19.8 \%$
\end{tabular}

Fifty percent of the evaluations are given to the left of the plus sign, and 50\% to the right. Both candidates have a $(50+$ 08.1)\% majority for [assez bien, assez bien]. But Macron has a $(50+09) \%$ majority for [assez bien, passable], while Mélenchon has a $(50+09) \%$ majority for [assez bien, assez bien]. That is why majority judgment (MJ) places Mélenchon above Macron: Mélenchon $\succ_{\mathrm{MJ}}$ Macron. In an analogous way, we obtain Mélenchon $\succ_{\mathrm{MJ}}$ Macron $\succ_{\mathrm{MJ}}$ Hamon $\succ_{\text {MJ }}$ Dupont-Aignan $\succ_{\text {MJ }}$ Le Pen.

\section{Axioms for Electoral Rules}

In [3], Balinski and Laraki consider the following axioms for an electoral rule $\mathrm{M}$ :

1. Basis of Comparison: The input consists of the individual preference orderings of the voters over the candidates. (This does not apply to majority judgment.)

2. Unrestricted Domain: Any vote configuration (profile) is allowed; i.e., the electoral rule should yield an output for every vote configuration as input.

3. Anonymity: Interchanging the names of the voters does not change the outcome.

4. Neutrality: Similarly, interchanging the names of the candidates does not change the outcome.

5. Monotonicity: If $A$ wins or is in a tie with $B\left(A \succcurlyeq_{\mathrm{M}} B\right)$ and one or more voters change their preference in favor of $A$, then the electoral rule will place $A$ above $B\left(A \succ_{\mathrm{M}} B\right)$.

6. Completeness: For each pair of candidates $A$ and $B$, the electoral rule places $A$ above $B\left(A \succ_{\mathrm{M}} B\right)$ or $B$ above $A$ $\left(B \succ_{\mathrm{M}} A\right)$ or declares $A$ and $B$ indifferent $\left(A \approx_{\mathrm{M}} B\right)$.

THEOREM 1 (May 1952) In the case of only two candidates, the only electoral rule that meets the above six axioms is majority rule (MR). (Recall that in the case of only two alternatives, majority rule amounts to the same thing as plurality rule.)

Proof. (Balinski and Laraki [3]) Suppose the electoral rule $\mathrm{M}$ satisfies all six axioms above. Anonymity implies that only the following numbers count: the number $n_{A}$ of voters who prefer $A$ to $B$, the number $n_{B}$ of voters who prefer $B$ to $A$, and the number $n_{A B}$ of voters who are indifferent

${ }^{1}$ Très bien = very good, bien = good, assez bien = fairly good, passable $=$ acceptable, insuffisant $=$ not good enough, à rejeter $=$ unacceptable . 
between $A$ and $B$. Completeness guarantees that there must be an outcome.

Suppose $n_{A}=n_{B}$ and $A \succ_{\mathrm{M}} B$. Because of neutrality, interchanging the names of $A$ and $B$ results in $B \succ_{\mathrm{M}} A$. But the new profile is identical to the original profile. Contradiction. Therefore,

$$
A \approx_{M} B \text { if } n_{A}=n_{B} .
$$

Suppose $n_{A}>n_{B}$. Change the preferences of $n_{A}-n_{B}$ voters who prefer $A$ to $B$ into indifferences, resulting in a valid profile in which the number of voters who prefer $A$ to $B$ equals the number of voters who prefer $B$ to $A$; according to axiom 2, all profiles are allowed. For this new profile, we have $A \approx_{\mathrm{M}} B$, because of $(*)$. By the monotonicity axiom, changing back to the original profile will yield $A \succ_{\mathrm{M}} B$.

For the case of any number of candidates, Balinski and Laraki add two more axioms to the above six:

7. Transitivity: If $A \succeq_{\mathrm{M}} B$ and $B \succeq_{\mathrm{M}} C$, then $A \succeq_{\mathrm{M}} C$.

As we shall see, majority rule (MR) is not transitive, while majority judgment is!

8. Independence of Irrelevant Alternatives (IIA): If $A \succeq_{\mathrm{M}} B$ and other candidates are added or removed, then we still have $A \succeq_{\mathrm{M}} B$.

As we shall see, majority rule is not transitive, and plurality rule is not IIA, while majority judgment, on the other hand, is both transitive and IIA.

We first show that majority rule (MR) is not transitive. Consider the following so-called Condorcet profile, showing three rankings each preferred by one-third of the electorate:

\begin{tabular}{|c|c|c|c|}
\hline & 1/3: & $A$ & $B$ \\
\hline & 1/3: & $B$ & C \\
\hline 1/3: & $C$ & & $B$ \\
\hline
\end{tabular}

The first and third groups, hence a majority, prefer $A$ to $B$, whence $A \succ_{\mathrm{MR}} B$. The first and second groups, hence a majority, prefer $B$ to $C$, whence $B \succ_{\mathrm{MR}} C$. But the second and third groups prefer $C$ to $A$, whence $C \succ_{\mathrm{MR}} A$. So, applied to this Condorcet profile, majority rule yields a cyclic outcome; there is no winner: $A \succ_{\mathrm{MR}} B \succ_{\mathrm{MR}} C \succ_{\mathrm{MR}} A$. This is called Condorcet's paradox. One might think that this problem does not occur in practice. However, under the impartial anonymous culture assumption (IAC), Gehrlein [9] showed that in the case of three candidates and a large number of voters, the probability that such a cyclic outcome occurs is about $1 / 16$.
Next we show that plurality rule is not IIA. Consider the following profile, which actually occurred in 2000 in the presidential election in Florida:

$$
\begin{array}{l|lll}
2,912,790 & 49.18 \%: & \text { Bush } & \\
2,912,253 & 49.17 \%: & \text { Gore } & \\
97,488 & 01.65 \%: & \text { Nader } &
\end{array}
$$

Under plurality rule (PR), Bush beats Gore: Bush $\succ_{\text {PR }}$ Gore. However, in the competition between Bush and Gore, a third (irrelevant) candidate, Nader, plays a decisive role: if Nader had not participated, (most) Nader voters would have voted for Gore, and Gore would have defeated Bush. Under plurality rule, a third, irrelevant, candidate determines the result in the competition between Bush and Gore!

Theorem 2 (Balinski and Laraki's version of Arrow's impossibility theorem) For three or more candidates, there is no electoral rule $M$ that satisfies axioms 1 through 8.

Proof (Balinski and Laraki [3]) Consider two arbitrary candidates $A$ and $B$. According to IIA, it is sufficient to consider only these two. Axioms 1 to 6 imply that the electoral rule $\mathrm{M}$ should be majority rule (MR). Because the domain is unrestricted, Condorcet's paradox shows that MR violates transitivity. Hence, there can be no electoral rule that satisfies all axioms 1 through 8 .

Arrow's original impossibility theorem [1] from 1951 states that in the case of three or more alternatives, any electoral rule that satisfies all eight axioms above, but with monotonicity replaced by Pareto's principle, is a dictatorship, i.e., there is a voter, called the dictator, such that the social outcome is always his preference ordering. In other words, the properties in question together with nondictatorship are logically inconsistent, i.e., they cannot be satisfied all at the same time. By Pareto's principle, we mean that if all voters weakly prefer $A$ to $B$, then also society should weakly prefer $A$ to $B$. By the way, while the proof of Arrow's impossibility theorem is relatively complicated, the proof of Balinski and Laraki's version of Arrow's theorem is relatively simple.

\section{Properties of Majority Judgment}

Axiom 1 above is now replaced with the following axiom:

$1^{*}$ : The input for an electoral rule consists of the evaluations (instead of the preference orderings) of the candidates by the voters. 
Then we have the following:

- Majority judgment (MJ) satisfies axiom $1^{*}$ and axioms 2 through 8. In particular, $\mathrm{MJ}$ is monotonic, transitive, and IIA. Keep in mind that no electoral rule can satisfy axioms $1,2, \ldots, 8$.

- Majority judgment (MJ) respects dominance. MR and PR do not!

- In addition, majority judgment (MJ) is proof against strategic manipulation with respect to the majority grade: a group with input higher (respectively lower) than the majority grade of a candidate cannot raise (respectively lower) the majority grade. For instance, suppose candidate $A$ gets the evaluations good, acceptable, poor. The majority grade of $A$ is then acceptable. The voter who gave him a good rating cannot raise $A$ 's majority grade: rating $A$ excellent instead of good does not change $A$ 's majority grade. This property certainly does not hold for point-summing systems; see the section below on the Borda rule, range voting, and approval voting.

- Majority judgment is partially proof against strategic manipulation with respect to the social ranking of the candidates: if a voter who prefers $B$ to $A$ (respectively $A$ to $B$ ) can raise (respectively lower) $B$ 's majority grade, then he cannot lower (respectively raise) $A$ 's majority grade; and if he can lower $A$ 's majority grade, then he cannot raise $B$ 's. For instance, suppose voter $i$ gives $B$ a higher evaluation than $A$, and $A$ has the same majority grade as $B$ (see the diagram below):

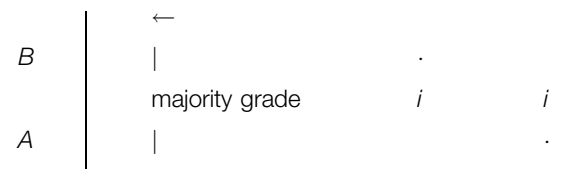

The only way in which voter $i$ may raise $B$ 's majority grade is by giving $B$ an evaluation higher than $B$ 's majority grade instead of a lower evaluation. But because $i$ gave $A$ a lower evaluation than that given to $B$, he cannot lower $A$ 's majority grade. Again, this property does not hold for point-summing methods.

- A candidate's majority grade is an important signal to the candidate and to the electorate. And majority judgment (MJ) stimulates candidates to get the highest possible evaluations of as many voters as possible; every grade (evaluation) contributes to the final judgment (the majority grade). And as remarked by Paul Edelman in [7], one may expect that "if all voters are routinely asked to grade, rather than rank, it is possible that a language and culture will develop that will reinforce objective behavior."

\section{Objections to Majority Judgment}

Even before the appearance of Balinski and Laraki's book [2], several examples had been given by various authors in which majority judgment at first sight gives a counterintuitive result; see, for instance, [7, 10, 15]. As with many paradoxes, although these examples may puzzle us, after some careful consideration, they frequently turn out to give us new insights. For instance, the liar's paradox, which has puzzled philosophers for more than two thousand years, was turned into the famous incompleteness theorem by Gödel in 1931. In Chapter 16 of their book [2], Balinski and Laraki take great pains to discuss many so-called paradoxical results in majority judgment and give an explanation for why these results on closer inspection are not paradoxical. Not everyone, however, will agree that their discussion and defense of the so-called paradoxical results are convincing.

Here is a typical example of a so-called paradoxical result: Suppose that $51 \%$ of the voters grade candidate $A$ as excellent and the other $49 \%$ grade $A$ as reject, while everyone (100\%) grades candidate $B$ as very good. Then $A$ 's majority grade will be excellent, and $B$ 's only very good. Hence, $A \succ_{\mathrm{MJ}} B$, while other voting methods may select $B$ as the winner. For instance, if we award a grade of excellent five points, while very good gets four, then $A$ will earn $51 \times 5=255$ points, while $B$ will earn $100 \times 4=400$. Balinski and Laraki would argue in this case that there is a majority that judges that $A$ deserves at least a grade of excellent, while there is no such majority for $B$. But we have been used for so long to think in terms of point summing and/or pairwise comparison that we would have to give up this familiar way of thinking and start thinking within a different framework. Notice also that if only $1 \%$ of the voters changed their evaluation of $A$ from excellent to very good, then $B$ would become the majority judgment winner, illustrating that a single vote may have far-reaching consequences. Majority judgment favors candidates who are capable of arousing the enthusiasm of a majority of the people. As computer simulations show [2, Chapter 19], point-summing methods favor centrist candidates more than majority judgment does.

A general argument of Balinski and Laraki's is that the majority decides on grades horizontally in the new model, just as it decides on the order between two competitors vertically in the traditional model. However, the vertical view completely ignores the information contained in the intensities of the evaluations. Majorities of grades are clearly considerably more discerning decisions than majorities of preferences. Why should this majority decision-reached by looking at the example horizontally-be any less valid than the traditional majority decision reached by looking at the example vertically?

For illustration, let us consider an example called the noshow paradox. Assume that five judges give the following evaluations of the two candidates $X$ and $Y$ in the table below:

\begin{tabular}{l|lllll}
$x$ & ex & vg & go & po & po \\
$y$ & ex & vg & ac & ac & po
\end{tabular}

Here $X$ 's majority grade is good (go), while $Y$ 's is only acceptable (ac); hence $X$ is the winner: $X \succ_{\mathrm{MJ}} Y$. Now a sixth judge shows up, who gives $X$ a higher grade than $Y$, 
say poor (po) for $X$ and reject (re) for $Y$, as in the table below:

\begin{tabular}{l|llllll}
$X$ & ex & vg & go & po & po & po \\
$y$ & ex & vg & ac & ac & po & re
\end{tabular}

Now we see that for $X$ there is a $4 / 6$ majority for [go, po], while for $Y$ there is a $4 / 6$ majority for [ac, ac]. Because neither of these two intervals dominates the other and because the consensus for $Y$ is greater than that for $X$, majority judgment now makes $Y$ the winner: $Y \succ_{\mathrm{MJ}} X$. One might conclude that the sixth judge would have done better not to participate, since he preferred $X$ (po) to $Y$ (re).

So majority judgment is not participant-consistent. It is also not join-consistent: $X$ wins in the five-judge electorate and in the one-judge electorate, yet does not win in the entire six-judge electorate. Finally, majority judgment does not cancel properly: suppose the sixth judge gives a rating of poor to both $X$ and $Y$; then again $Y$ becomes the winner.

Balinski and Laraki point out that the sixth judge seems to have a low opinion of both candidates, may not care much who wins, and may be pleased to see their majority grades lowered. In addition, if he felt strongly about preferring $X$ to $Y$ and gave $X$ a grade between excellent and good and $Y$ a grade between acceptable and reject, then $X$ would remain the winner. Clearly, majority judgment gives to every voter the possibility of altering the ranking, whether or not one is indifferent between several or all candidates. This is a clear inducement to participate, which is not true of point-summing methods that do cancel properly [2, p. 287].

In [2, Section 4.3], Balinski and Laraki point out that the three properties participant-consistency, join-consistency, and proper cancellation are precisely the three properties that every rule that is consistent with Condorcet-i.e., selects the Condorcet winner if it exists-fails to satisfy in the traditional model. In addition:

If a candidate moves up in the estimation of the voters, then she should not lose in the final standings (choice-monotonicity). If voters' estimations remain the same except that the winner moves up, then not only should she still be the winner but the final ranking among all the others should remain the same (rank-monotonicity). Some methods of the traditional model satisfy one or the other of these two properties, but none satisfy both (theorem 4.4). And no method in the traditional model guarantees that when a nonwinner falls in the estimation of the voters, the winning candidate remains the winner (strong monotonicity, chapter 5). The majority judgment is at once choice-monotonic, rank-monotonic and strongly monotonic [2, Section 16.3].

Edelman argues that the traditional approach to election design focuses solely on the best method to aggregate the preferences or evaluations of the voters:

But elections are run by institutions, and the interests of the institution may not be reflected in the preferences of the voter [8, p. 287].
Borda Rule, Range Voting, and Approval Voting

The question whether it is possible to escape from Arrow's theorem has puzzled many economists, mathematicians, political scientists, and philosophers for about 50 years. In 1998, Donald G. Saari [12] pointed out that the IIA condition has the devastating effect that the information that the voters are rational, i.e., that they have transitive preferences, is completely ignored: IIA says that the voting procedure has only to consider pairs of alternatives, but transitivity is a condition on triples of alternatives. In other words, IIA prevents the electoral rule from taking voters' ability to think transitively into account, and hence we cannot expect a rational (transitive) output. Saari concludes that if society wants rational outcomes, it must avoid procedures intended for irrational voters, i.e., IIA is not a reasonable condition to impose on an electoral rule meant for rational voters. As Saari explains, this would be similar to requiring that the function $f$ defined by $f(x)=\frac{1}{x-4}$ have an unrestricted domain. Saari's solution is to replace IIA by a condition that does not eliminate the information characteristic of rational voters, namely, that their preferences are transitive:

Definition 3 (Intensity independence of irrelevant alternatives (IIIA)) In intensity independence of irrelevant alternatives, the social ranking of a pair depends on the relative rankings of this pair by the voters and their intensity levels of this ranking, where the intensity level of a pair $\{a, b\}$ is the number of candidates that separate $a$ and $b$. For instance, if a voter has individual ranking $a \succ c \succ d \succ b$, then the intensity level of the pair $\{a, b\}$ is 2 .

We have seen that plurality rule and majority rule do not take into account the intensities by which voters prefer one candidate to another. However, the Borda rule (BR) does take these intensities into account in a particular way: if there are four alternatives $a, b, c, d$ and a voter has preference order $a \succ b \succ c \succ d$, then $a$ receives three Borda points from this voter, because $a$ beats three other candidates; $b$ gets two Borda points because it beats two other candidates; $c$ gets one Borda point, and $d$ gets zero. And the Borda score of an alternative is the sum of the Borda scores of this alternative over all voters. And indeed, the Borda rule (BR) satisfies IIIA, although it does not satisfy IIA.

Nevertheless, although the Borda rule does in some (restricted) sense take intensities of preference into account, it remains true that if two voters have the same preference order $A \succ B \succ C$, one voter may mean that he judges $A$ as excellent, $B$ as very good, and $C$ as good, while the other voter may mean that he judges $A$ as excellent, $B$ as poor, and $C$ as reject. In both cases, $A$ 's Borda score is 2, $B$ 's is 1 , and $C$ s is 0 . But the intensities by which the voters prefer $A$ to $B$ and $B$ to $C$ are very different.

Because the Borda rule coincides with plurality and majority rule in the case of only two alternatives, it also does not respect dominance.

The following example illustrates that the Borda winner may be the majority judgment loser. Suppose seven voters 
have the following preferences over three alternatives $A, B$, C:

\begin{tabular}{l|lll}
3 voters: & $A(\mathrm{ex})$ & $B(\mathrm{po})$ & $C(\mathrm{re})$ \\
2 voters: & $B(\mathrm{ex})$ & $C(\mathrm{vg})$ & $A(\mathrm{go})$ \\
2 voters: & $C(\mathrm{ex})$ & $B(\mathrm{ac})$ & $A(\mathrm{po})$
\end{tabular}

Then B's Borda score is $3+4+2=9, C$ s $s$ is $0+2+4=6$, and $A$ 's is $6+0+0=6$. So the Borda ranking is $B \succ_{\mathrm{BR}} C \approx_{\mathrm{BR}} A$. It may sound very plausible that $B$ is the Borda winner, because for every voter, $B$ is their first or second choice. However, this may be misleading when second choice means only poor or acceptable, as in our example. Look at the evaluations received by the candidates as indicated above, ordered from high to low:

\begin{tabular}{l|lllllll}
$A$ & ex & ex & ex & go & go & po & po \\
$B$ & ex & ex & ac & ac & po & po & po \\
$C$ & ex & ex & vg & vg & re & re & re
\end{tabular}

We see that $C$ s majority grade is very good, $A$ 's is good, and $B$ 's is only acceptable. Therefore, we have $C \succ_{\mathrm{MJ}} A \succ_{\mathrm{MJ}} B$. Also notice that the evaluations of $A$ dominate those of $B$, while $B$ was the Borda winner.

A well-known method to take into account the intensities by which voters prefer one candidate to another is score voting, also called range voting, as promoted by Warren D. Smith in [13]. Each vote consists of a numerical score within some range, e.g., 0 to 99 or 0 to 9 . Voters may also indicate no opinion if they wish to express no opinion about a candidate; such votes do not affect that candidate's average score. The candidate with the highest average score wins. Although this method has many nice properties (see [13]), it is not proof against manipulation: if my honest score for candidate $A$ is 7 on a scale of 0 to 9 and I prefer candidate $A$ to its competitor $B$, who in my opinion deserves a 5 , I may dishonestly give my favored candidate $A$ a 9 and at the same time its close competitor $B$ a 1 and in this way both increase $A$ 's average score and decrease $B$ 's. While range voting ranks the candidates or alternatives by their mean scores or ratings, majority judgment does so by their median ratings and in this way restricts the possibilities for successful manipulation, as has been explained above.

In the context of parliamentary elections, notice that majority judgment may be used to determine a ranking of the different parties, but that it is not appropriate for determining a seat distribution among the different parties. To do the latter, one could ask voters to give each party a grade between 0 and 9, add the total number of grades for each party, and divide the seats in proportion to these sum totals. This idea is elaborated in [16]. In the Dutch education system, the grades 0 to 9 are familiar to both students and teachers and therefore have a (relatively) clear meaning for everyone. In the French education system, the grades 0 to 20 are used.

Notice that every method by which voters give points to candidates and in which candidates are ranked according to the number of points they have collected also satisfies axioms $1^{*}$ and 2 through 8 . But such a method is not proof against strategic manipulation with respect to the final judgment about a candidate nor with respect to the ranking of the candidates. In addition, any method based on summing or averaging points is consistent with neither majority judgment (MJ) nor majority rule (MR). Consider, for instance, the following profile:

\begin{tabular}{l|rrrrrrr} 
& $\mathbf{1}$ & $\mathbf{2}$ & $\mathbf{3}$ & $\mathbf{4}$ & $\mathbf{5}$ & $\mathbf{6}$ & $\mathbf{7}$ \\
\hline$A$ & ex & ex & ex & ac & ac & ac & ac \\
$B$ & po & po & po & go & go & go & go
\end{tabular}

Looking at this profile horizontally, we see that $B \succ_{\mathrm{MJ}} A$ (good vs. acceptable), and looking vertically yields $B \succ_{\mathrm{MR}}$ $A$ (four preferences to three). But with 5, 4, 3, 2, 1, 0 points for, respectively, ex, vg, go, ac, po, re, $A$ defeats $B, 23$ points to 15 . Hence, summing or averaging points is consistent with neither majority judgment (MJ) nor majority rule (MR).

The idea of approval voting is that every voter gives one point to the candidates of which he approves and zero points to the candidates of which he disapproves. Consequently, approval voting is in fact majority judgment with only two grades: acceptable and reject. However, as we have stressed before, the language of grades should be sufficiently rich that every voter who wants to distinguish two candidates is able to express this in the given language of grades.

Let us consider again the profile given above. With one point (approval) for good or higher, $B$ wins four points to three for $A$. But with one point (approval) for acceptable or higher, $A$ beats $B$ seven points to four. Hence, approval voting gives arbitrary outcomes and is consistent with neither majority judgment (MJ) nor majority rule (MR).

Also notice that approval voting (AV) does not respect dominance. For instance, consider the following merit profile for $A$ and $B$ :

\begin{tabular}{l|lllllll}
$A$ & ex & ex & ex & ex & po & po & po \\
$B$ & go & go & go & go & re & re & re
\end{tabular}

Clearly, $A$ dominates $B$, and we have $A \succ_{\mathrm{MJ}} B$, but if approval means good or better, then $A \approx_{\mathrm{AV}} B$. For more on majority judgment versus approval voting, see [6].

\section{Summary}

Asking voters for their preference ordering over a group of candidates (let alone asking them for only their first choice) is asking for trouble, resulting in a Babylonian confusion of tongues: different voters may say the same words (saying they prefer $A$ to $B$ and $B$ to $C$ ), while meaning completely different things. Hence, Arrow's impossibility theorem (1951) comes as no big surprise: any voting method that takes the preference orderings of voters as input and satisfies independence of irrelevant alternatives (IIA) and some other little-disputed conditions is doomed to result in a dictatorship. One solution, put forward by Saari [12], is to weaken the condition of IIA, 
since it in fact causes the voting rule to ignore the information that the voters are rational, i.e., have transitive preferences. The Borda rule satisfies this weakened IIA condition.

The topic of this paper is another solution proposed by Balinski and Laraki [2], namely, to ask voters for a different input: instead of giving their individual preference orderings over the candidates, they are asked to give their evaluations of the candidates in a sufficiently rich language of grades. Then it also becomes clear that plurality rule, majority rule, and the Borda rule do not respect dominance, i.e., the candidate with the better evaluations may not be the winner.

Asking voters for their evaluation of the candidates gives much more information and-essentially by taking the median value of the candidate's evaluations-majority judgment (MJ) aggregates this extra information in an adequate way to a social ranking of the candidates, which renders the global support that candidates get from the electorate.

Majority judgment (MJ) is transitive and independent of irrelevant alternatives (IIA); in addition, it respects dominance and is strategy proof in grading (but only partially in ranking). Majority rule (MR) is IIA, but not transitive, while plurality rule (PR) and the Borda rule (BR) are transitive but not IIA. None of PR, MR, and BR respects dominance: the candidate with the better evaluations may not be the winner.

Any method based on summing or averaging points is inconsistent with majority judgment and with majority rule. Approval voting (AV) has too few grades, and consequently, it gives arbitrary outcomes and is consistent with neither majority judgment nor majority rule. Also, approval voting does not respect dominance.

The electoral system in the USA does not respect dominance. The French electoral system is not monotonic: by getting more support (in the first round), a candidate may lose rather than win the presidency (in the second round).

\section{ACKNOWLEDGMENTS}

I would like to thank several anonymous referees and editor-in-chief Sergei Tabachnikov for suggesting important improvements to earlier versions of this paper.

\section{OPEN ACCESS}

This article is licensed under a Creative Commons Attribution 4.0 International License, which permits use, sharing, adaptation, distribution and reproduction in any medium or format, as long as you give appropriate credit to the original author(s) and the source, provide a link to the Creative Commons licence, and indicate if changes were made. The images or other third party material in this article are included in the article's Creative Commons licence, unless indicated otherwise in a credit line to the material. If material is not included in the article's Creative Commons licence and your intended use is not permitted by statutory regulation or exceeds the permitted use, you will need to obtain permission directly from the copyright holder. To view a copy of this licence, visit http://creativecommons.org/licenses/by/4.0/.
Harrie de Swart

Department of Philosophy

Tilburg University

Warandelaan 25037 AB Tilburg

The Netherlands

e-mail: h.c.m.deswart@tillburguniversity.edu

\section{REFERENCES}

[1] K. Arrow. Social Choice and Individual Values. Yale University Press, 1951.

[2] M. Balinski and R. Laraki. Majority Judgment; Measuring, Ranking and Electing. MIT Press, 2010.

[3] M. Balinski and R. Laraki. Majority judgment vs majority rule. Social Choice and Welfare 2019. Available at https://doi.org/10. 1007/s00355-019-01200-x.

[4] M. Balinski and R. Laraki. Trump and Clinton victorious: proof that US voting system does not work. The Conversation 58752, 2016.

[5] M. Balinski and R. Laraki. Pour éviter un nouveau 21 Avril instaurons le jugement majoritaire. The Conversation 58178, 2016.

[6] M. Balinski and R. Laraki. Majority judgment vs. approval voting. CREST, Srie des Documents de Travail, 2018-15. Operations Research 2020.

[7] P. H. Edelman. Review of Michel Balinski and Rida Laraki's book [2] on majority judgment. Public Choice 151 (2012), 807-810.

[8] P. H. Edelman. The institutional dimension of election design. Public Choice 153 (2012), 287-293.

[9] W. V. Gehrlein. Condorcet's paradox and the likelihood of its occurrence: different perspectives on balanced preferences. Theory and Decision 52 (2002), 171-199.

[10] Dan S. Felsenthal and M. Machover. The majority judgement voting procedure: a critical evaluation. Homo Oeconomicus 25 (2008), 319-334.

[11] K. O. May. A set of independent, necessary and sufficient conditions for simple majority decision. Econometrica 20 (1952), 680-684.

[12] D. G. Saari. Connecting and resolving Sen's and Arrow's theorems. Social Choice and Welfare 15 (1998), 239-261.

[13] Warren D. Smith. Range Voting. Available at https://rangevoting. org.

[14] Harrie de Swart. Philosophical and Mathematical Logic. Springer, 2018.

[15] M. A. Zahid and $\mathrm{H}$. de Swart. The majority judgment theory and paradoxical results. International Journal of Arts and Sciences 4:20 (2011), 121-131.

[16] M. A. Zahid and $H$. de Swart. Experimental results about linguistic voting. Czech Economic Review 9 (2015), 170-187.

Publisher's Note Springer Nature remains neutral with regard to jurisdictional claims in published maps and institutional affiliations. 